\title{
Novel Concepts for Surface Movement Radar Design
}

\author{
Konstantin Lukin, Senior Member, IEEE, Anatoliy Mogila \\ LNDES, Institute for Radiophysics and Electronics NAS of Ukraine \\ 12 Akad. Proskura St., Kharkov, 61085, Ukraine \\ Tel.+38 057 7203349, Fax +38 057 3152105; Email: LNDES@kharkov.com; lukin@,ire.kharkov.ua \\ Gaspare Galati, Senior Member, IEEE, Gabriele Pavan \\ DISP, Department of Computer Science, System and Production \\ Tor Vergata University, Roma, Via del Politecnico, 1 - 00133 Roma Italy \\ Tel.+39 06 72597417, Fax +39 06 72597532; Email: galati@disp.uniroma2.it; pavan@disp.uniroma2.it
}

\begin{abstract}
Traditional millimeter wave Surface Movement Radar (SMR) have been designed and tested. A novel concept for the design of a new SMR is suggested based upon Synthetic Aperture Antenna and Noise Radar Technology. It enables designing of SMR without mechanical rotation of an antenna, but applying several nonmoving Synthetic Aperture Antennas instead. Application of Noise Radar Technology is also considered in more detail to improve the expected performance of a new SMR.
\end{abstract}

Index Terms-Surface Moving Radar, Noise Radar, ASMGCS

\section{INTRODUCTION}

A network of Surface Movement Radar (SMR) is normally used as the basis for Advanced-Surface Movement Guidance and Control System (A-SMGCS) design for large airports [1]. Various types of radar have been suggested as candidates for SMR following both the main requirements of such systems and the capabilities of the current radar developments.

In Section II we describe the A-SMCGS functions and the SMR requirements [3]. Most of the current SMR works at $\mathrm{X}$-band, this implies the use of large antennas, with a rotation speed of $60 R P M$. In many practical situations the airport surface to be covered is limited to the runways and their neighbouring areas within an angle around $180^{\circ}$. Therefore the new idea is to substitute the rotating antenna in conventional SMR by a set of nonmoving Synthetic Aperture Antennas (SAA's) to cover a defined area. The number of SAA's to be used depends on the area to be covered.

In Section III we present the main idea [6] and formulate general requirements to its basic elements. In Section IV we give a detailed description of three types of the Synthetic Aperture Antenna developed in LNDES IRE NASU [6], [7] which enables avoiding of rotating antennas which are usually used in SMRs.

In Section $\mathrm{V}$ we also present results of theoretical and experimental evaluation of potential capabilities of Noise Radar Technology (NRT) [8], [10] for SMR design and development.

\section{A-SMCGS FunCTIONS AND REQUIREMENTS IN SMR}

The capacity of an A-SMGCS is defined as the maximum number of simultaneous movements of aircraft and vehicles that the system can safely support within an acceptable delay related to the runway and taxiway capacity of a particular airport. The A-SMGCS provides the following functions (as described in Table I where the functions are compared to SMGCS): surveillance, control, guidance and routing/planning to aircraft and vehicles in order to keep up the airport capacity under all local weather conditions, while maintaining the required level of safety.

Table I - Comparison functions of SMGCS with A-SMGCS.

\begin{tabular}{|l|l|l|}
\hline Functions & SMGCS & A-SMGCS \\
\hline Surveillance & $\begin{array}{l}\text { By visual observation } \\
\text { (radar-assisted in } \\
\text { poor visibility) }\end{array}$ & $\begin{array}{l}\text { Location and identification of } \\
\text { mobiles and obstacles; } \\
\text { sensors and data fusion }\end{array}$ \\
\hline Control & $\begin{array}{l}\text { Manual/semiautoma- } \\
\text { tic, by controller } \\
\text { understanding }\end{array}$ & $\begin{array}{l}\text { Automatic, with conformance } \\
\text { monitoring, conflict analysis } \\
\text { and resolution (mainly } \\
\text { against Runway incursions) }\end{array}$ \\
\hline Guidance & $\begin{array}{l}\text { By ground-based } \\
\text { (mainly fixed) } \\
\text { visual aids }\end{array}$ & $\begin{array}{l}\text { Automatic, using improved } \\
\text { visual aids (variable signs, } \\
\text { moving lights) and possibly } \\
\text { cockpit display with data link }\end{array}$ \\
\hline $\begin{array}{l}\text { Routing/ } \\
\text { Planning }\end{array}$ & $\begin{array}{l}\text { Manual, by } \\
\text { controller reasoning }\end{array}$ & $\begin{array}{l}\text { Automatic, with optimal } \\
\text { definition and assignment of } \\
\text { push-back and start-up time, } \\
\text { departure sequence, taxi } \\
\text { routes }\end{array}$ \\
\hline
\end{tabular}

SMR is the main sensor in A-SMGCS and it must have the following characteristics [1], [2]:

1. High accuracy and resolution capabilities in range and azimuth measurements on the entire airport surface so as to recognize the shape and the orientation of the target (features extraction and classification/identification). The system shall be able to detect objects (whether fixed or mobile) whose radar cross section is $1 \mathrm{~m}^{2}$ (typical value for the X-band) with a $90 \%$ probability even in severe weather (e.g. with a rainfall rate up to $16 \mathrm{~mm} / \mathrm{h}$ ). The resolution shall be better than $10 \mathrm{~m}$ (aiming at a target value of 3-6 m) and anyway such to allow for subsequent image processing in order to assess both the size and the orientation of the vehicle.

2. The coverage area has to be large enough to overlap the airport surface (runways, taxiways, Apron). Single radar can have problem with shadowing, therefore a Multi 
Radar Network is an advanced solution for future systems.

3. The high resolution imposes a pulse width of about 40 nanoseconds and an antenna mainlobe width less than $0.4^{\circ}$ (desirable values: $20 \mathrm{~ns}, 0.2^{\circ}$ ).

4. The data refreshing period shall not be greater than $1 \mathrm{~s}$.

5. The system shall be able to track up to 400 objects at the same time, with a processing delay (latency) lower than $100 \mathrm{~ms}$.

6. The object location shall be measured with a precision better than $5 \mathrm{~m}$. Velocity shall be measured for both cooperating and non-cooperating targets with accuracy better than $2.5 \mathrm{~km} / \mathrm{h}$ for speeds in the range $5-100 \mathrm{~km} / \mathrm{h}$.

7. The system shall be able to visualize small-sized objects (such as suitcases), which can accidentally lie on the ground.

8. The installation of the antenna and of the radar shall be as easy as possible. Low microwave (or millimeter wave) transmitted power (small sized-antennas) reduce the installation requirements and cost.

9. Electromagnetic compatibility has to be guaranteed with radars of the network and other radar and communication systems in the airport.

10. Full respect of ICAO and EUROCAE standards and integration purposes in A-SMGCS system [3].

Main problems in SMR are: (i) control of false alarms, especially in clutter; (ii) rain attenuation at highest frequencies; (iii) plot extraction [4], as aircraft targets have extension up to $60-70 \mathrm{~m}$ as compared to radar resolution cells of $6 m \times 0.4$ degrees $(3 m \times 0.2$ degrees in high resolution radar) and occupy hundreds of resolution cells. The resulting "target splitting" is a well-known phenomenon in SMGCS. Finally the Constant False Alarm Rate (CFAR) threshold is a key element of the plot extraction process. It must guarantee a limited detection loss (e.g., below $2 d B$ ) and satisfy the conflicting requirements of detecting: (a) both extended targets (e.g. aircraft), and small targets (e.g. persons, small vehicles); (b) both fixed and moving targets; (c) in small, fixed clutter environment; in medium, slowly varying clutter and in strong, quickly varying clutter.

The above requirements can be met with a network of lightweight, compact, millimeter-wave (W-band, $95 \mathrm{GHz}$ ) radars [1]. The millimeter-wave band has the following advantages:

- Reduced equipment size and simpler installation.

- Easy integration in an existing airport traffic control system.

- Low average transmitted power.

- Larger radar cross-section (RCS).

- Detection of any kind of targets.

- Detection and imaging of aircraft (bearing angle extraction).

A first demonstrator of SMR working at W-band was developed early in 1995 in Oerlikon Contraves Italiana (OCI) and was tested by the end of 1996. Two identical radars have been operating for many months in Frankfurt airport and in Venice airport, respectively. The basic characteristics of the $\mathrm{W}$ - band radar are shown (nominal values) in Table II in comparison with other systems operating at $\mathrm{X}$ and $\mathrm{Ku}$ band.

The evaluation of the coverage performance of the W-band miniradar network has been done for several Italian and
European airports [5]. The number of miniradar sensors necessary to perform complete coverage of the airport layouts varies from 1 to 4 with a typical value of 2 for a medium-size airport such as Marco Polo in Venice.

Table II - Main characteristics of SMR.

\begin{tabular}{|c|c|c|c|c|}
\hline & $\begin{array}{c}\text { W-band } \\
\text { Miniradar }\end{array}$ & $\begin{array}{c}X \text {-band } \\
\text { N. } 1 \\
\end{array}$ & $\begin{array}{c}X \text {-band } \\
N .2\end{array}$ & $\begin{array}{c}\text { Ku-band } \\
\text { Radar }\end{array}$ \\
\hline $\begin{array}{c}\text { Frequency } \\
(\mathrm{GHz})\end{array}$ & $95 \mathrm{GHz}$ & $\begin{array}{c}9.375 \text { standard } \\
9.410 \text { standard } \\
9.170 \text { optional } \\
9.438 \text { optional } \\
9.490 \text { optional } \\
(*) \\
\end{array}$ & $\begin{array}{c}9.34-9.52 \\
\text { with } \\
\text { frequency } \\
\text { diversity }\end{array}$ & $15.7 \div 16.7$ \\
\hline $\begin{array}{c}\text { Pulse width } \\
\text { (ns) }\end{array}$ & 20 & 40 & 40 & 40 \\
\hline Azimuth & $0.2^{\circ}$ & $<0.37^{\circ}$ & $0.45^{\circ}$ & $0.33^{\circ}$ \\
\hline Elevation & $\begin{array}{c}\text { inverted } \\
\text { cosec }^{2}\end{array}$ & $\begin{array}{c}11^{\circ} \\
\text { inverted cosec }^{2}\end{array}$ & $\begin{array}{c}<18^{\circ} \\
\text { inverted } \\
\text { cosec }^{2} \\
\end{array}$ & $\begin{array}{c}23^{\circ} \\
\text { inverted } \\
\text { cosec }^{2}\end{array}$ \\
\hline $\begin{array}{c}\text { Trans. } \\
\text { Power }(k W) \\
\end{array}$ & 1.5 & 17 & 25 & $>20$ \\
\hline$P R F(H z)$ & $\begin{array}{c}4096 \\
\text { or integer } \\
\text { multiple } \\
\text { (up to 7) }\end{array}$ & $\begin{array}{c}\text { Programmable } \\
800-8000\end{array}$ & 10000 & 8192 \\
\hline $\begin{array}{l}\text { Ant. Rotation } \\
\text { Speed (RPM) }\end{array}$ & $\begin{array}{c}60 \\
\text { (selectable } \\
120)\end{array}$ & 60 & 60 & 60 \\
\hline $\begin{array}{c}\text { Antenna } \\
\text { Gain }(\mathrm{dB})\end{array}$ & 52 & $>38$ & 37 & 43 \\
\hline Polarization & Circular & Circular & Circular & Circular \\
\hline $\begin{array}{c}\text { Horizontal } \\
\text { Dimension } \\
(m)\end{array}$ & $1.1 \times 1.1$ & $<6.6$ (array) & 6 (array) & $\begin{array}{c}5.18 \\
\text { (radome) } \\
\approx 4 \\
\text { (antenna) }\end{array}$ \\
\hline Weight (kg) & 150 & $<375$ & - & - \\
\hline$R \max (k m)$ & $\begin{array}{c}3 \text { (clear) } \\
2 \text { (fog) } \\
1.5(\text { rain } \\
16 \mathrm{~mm} / \mathrm{h}) \\
(* *)\end{array}$ & $\begin{array}{c}5.5 \\
\left(P_{D}=0.99\right) \\
(* * *)\end{array}$ & $\begin{array}{c}6 \text { (clear) } \\
5.7 \text { (rain } 16 \\
\mathrm{~mm} / \mathrm{h}) \\
(* *)\end{array}$ & $\begin{array}{c}5.5 \text { (clear) } \\
4.5(\mathrm{rain} \\
16 \mathrm{~mm} / \mathrm{h}) \\
(* * * *)\end{array}$ \\
\hline
\end{tabular}

Notes:

(*) Standard uses magnetron of $25 \mathrm{~kW}$; optional employs magnetron of $30 \mathrm{~kW}$;

(**) A RCS of $1 \mathrm{~m}^{2}$ has been supposed at X-band, $10 \mathrm{~m}^{2}$ at W-band; $(* * *)$ In bad weather condition Rmax is unknown; $(* * * *) R C S$ of $2 \mathrm{~m}^{2}$.

\section{A NEW APPROACH TO SMR DESIGN}

As it may be seen from the above points $1-10$, Table I and Table II, there are many challenges in A-SMGCS design if one wants to use convention radar approach. In particular it is rather difficult to achieve simultaneously high range resolution and environmentally safe performance of SMR with working range from $200 \mathrm{~m}$ to $5000 \mathrm{~m}$ to be used in ASMGCS. On the other hand, a low cost of SMR required is difficult to combine with desirable flexibility of radar performance which may enhance operation and performance efficiency of the radar, coherent processing of radar returns and clutter suppression. High azimuth resolution requires relatively large dimension of the radar antenna aperture to be rotated with $60-120 R P M$. The idea to shortening of the radar signal wavelength faced the problem of high rate attenuation of the signal under raining or snowing weather conditions. For instance at W-band $(95 \mathrm{GHz})$ experimental results, [13], show a range coverage up to $3000 \mathrm{~m}$ in clear weather, $2000 \mathrm{~m}$ with $50 \mathrm{~m}$ fog visibility, better than $1200 \mathrm{~m}$ in rain (at the rainfall rate of $16 \mathrm{~mm} / \mathrm{h}$, as from EUROCAE/ICAO specifications and recommendations). 
Many challenges in design of efficient SMR may be solved if to apply novel concept suggested in [12] which in general is based upon the application of Ground Based Synthetic Aperture Radar (GB SAR) with high rate of SAR image generation for real time surveillance of an airport surface area. This idea may be implemented with the help of a new antenna named as Synthetic Aperture Antenna (SAA) [6], [7] in combination with SAR imaging technique and Noise Radar Technology [8], [9]. Fast motion of a resonant radiating slot along a real aperture is a distinguishing feature of the SAA suggested. Section IV describes SAA in more detail. This approach enables avoiding usage of the rotating antenna of conventional SMR and applies, instead, a set of static antennas, such as SAA, to cover the area of interest. The number of the SAAs to be used depends on the area to be covered (basically, it's azimuthally extent) and on the desirable/available power of the transmitted signal. Figure 1 shows schematically an example of such coverage for half plane area using four SAAs.

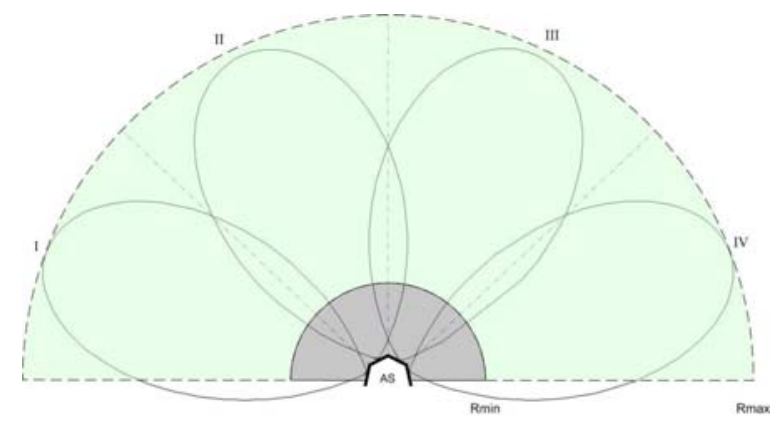

Figure 1 - Coverage of the airport area with four Synthetic Aperture Antennae.

However, depending on the working range and azimuth resolution required, the area of interest may be covered using a different number of the SAAs. We have evaluated for instance, that in case of not high azimuth resolution $(5 \mathrm{~m}-$ $10 \mathrm{~m}$ ) at 3 kilometres range and viewing angle of 90 degrees one may use one or two SAA's. On the other hand in case of shorter range, but wider viewing angle, one has to apply up to four antennae to get better azimuth resolution and keep the transmitted average power below $5 W$ in Ka-band. Many other options of SMR may be designed with performance dependent on the area to be monitored, working range and azimuth resolutions. Below we describe realistic ways of this concept implementation for two different options.

\section{Synthetic Aperture Antenna Techniques}

The basic idea of the suggested SAA consists in the following [6]. For antenna beam forming and scanning, we use the principles of 1D antenna array (AA), but with radiation/reception of electromagnetic pulses at each position of a single, moveable radiating/receiving element rather than simultaneous radiation/reception by all elements of the 1D-AA, as usually. In other words, we use the concept of synthetic aperture radar being applied to the 1DAA aperture. Generally, this approach enables application of both various types of radiating elements and methods for its motion implementation along the antenna aperture. The following parameters are of major interest: antenna beam width; number of beam positions; antenna pattern sidelobes and time of full scan. In the scanning antenna suggested, the beam width is defined by its real aperture, while the number of beam positions is defined by that of measurement positions for the radiating element. The sidelobes level will depend on the phase-amplitude distribution (weighting function) along the real aperture of the antenna. Finally, the time of full scan is defined by both the radiating element shift time to a neighbouring position and the numbers of those positions. The above shift-time in the antenna suggested may be done so short, that in real applications it should be increased to provide the required data acquisition time at each position if transmitted power is not sufficient. It is obvious, that for implementing a full scan in real time scale one has to perform both range and azimuth compressions in quasi-real time scale, as well. For instance, for $30 \mathrm{~m} / \mathrm{s}$ velocity, a typical speed of surface traffic, one azimuth resolution cell of $6 \mathrm{~m}$ will be passed by $0.2 \mathrm{~s}$, which gives possibility to upgrade the frame up to 5 times and enables watching smooth motion of such an object.

We have designed three types of Synthetic Aperture Scanning Antennas.

The first type of SAA uses realization of straight-line motion of radiating slot as shifts of cross-point of a straight line and a helical line when the latter is rotated. Helical-Slot SAA (HS-SAA) is made of two hollow co-axial cylinders with the minimal backlash between their surfaces. The outer cylinder has a longitudinal slot parallel to the cylinder axis, while the inner contains a helically shaped waveguide with either a single longitudinal slot or a set of half-wavelength slots properly oriented with respect to the slot of the outer cylinder. When rotating the inner cylinder, the cross-point of the longitudinal slot and helical waveguide will be moving along the slot. In this way, we implement linear motion of a single radiating element with the radiation suppression by others half-wavelength slots. Scanning rate of the antenna is defined by rotation rate of the inner cylinder and may be rather high when the radar has a properly fast SAR processor. The version of such scanning antennas has been designed in LNDES, IRE NASU and tested jointly with AeroSensing Radar System, GmbH [6]. Some details of its design are shown in Figure 2. The inner cylinder is referred to as the HS-SAA rotor while the outer cylinder is referred to as the HS-SAA stator. The latter contains a longitudinal slot supplied with 2D-horn: plane-parallel waveguide with smoothly widening cross-section. A helix formed rectangular waveguide (HFRW) is incorporated into the rotor body, Figure $2 \mathrm{~b}$. In the HFRW narrow wall, the rectangular resonant slots are cut with the required period, $\delta z$ and parallel to the $X$ axis. Hence, when rotating the rotor, the HFRW slots will be serially overlapping with the slot of the 2D-horn. The overlapping of the slots will occur at equally spaced positions along the $X$ axis, at the consecutive moments of time $t_{n}$. The period of the HFRW slots spacing is chosen to provide radiation of waveguide wave through the only slot in a time. To perform radar returns acquisition and further signal processing aiming the beam scanning realization the transceiver of the synthetic aperture radar is to be connected to the HFRW via waveguide rotary joint. The resonant slots in the HFRW narrow wall may excite a parasitic propagating wave inside the backlash between the rotor and stator of the antenna, which, in fact, is a slightly curved plane-parallel waveguide. In order to prevent excitation of that wave short circuit grooves (chokes) are to be made in the rotor body along 
both sides of the HFRW. Excitation of the 2D-horn will take place not only at the moments of symmetrical overlapping of the HFRW resonant slots and the slot of the 2D-horn, but also within some range of their mutual shift. In the antenna suggested, the radiation of probing pulses and reception of radar returns occur for different positions of resonant slots equally spaced by spatial period $\Delta x$ at the consecutive moments of time $t_{n}=(n+N) \Delta y /(R \Omega)$, where $\Omega$ is angular frequency rotor spinning; $\Delta y=\Delta x \operatorname{tg} \alpha$ is spacing between resonant slots along $Y$ axis; $R$ is the rotor radius; $\alpha$ is the helix angle; $n=-N, \ldots-1,0,1, \ldots . N$ and $2 N+1$ is the number of the resonant slots. The real aperture equals $L=2 N \Delta x$. Main dimensions of the designed HS-SAA shown in Figure 2 are as follows: the helix angle: $14.5 \mathrm{deg}$; the length and the width of resonant radiating slots: $4.8 \mathrm{~mm}$ and $0.8 \mathrm{~mm}$ respectively; spatial period along $X$ axis and $Y$ axis are $\Delta x=7 \mathrm{~mm}$ and $\Delta y=1.8 \mathrm{~mm}$, respectively; slot spacing along vertical axis: $1 \mathrm{~mm}$. With this dimensions and $97 \mathrm{~mm}$ diameter of the rotor one may perform linear shift of $51(N=25)$ radiating slots along the $400 \mathrm{~mm}$ aperture for half of full rotation of the antenna rotor. The rotation may be performed with a speed up to 2000 RPM if required. However in order to have 24 frames per second upgrade rate one has to rotate this antenna with spinning velocity of 720 RPM. This gives possibility to perform one full scan for 40 $m s$. This gives about $0.8 \mathrm{~ms}$ to switch radiating slot and about $0.4 \mathrm{~ms}$ for transmission and reception of the radar signal.
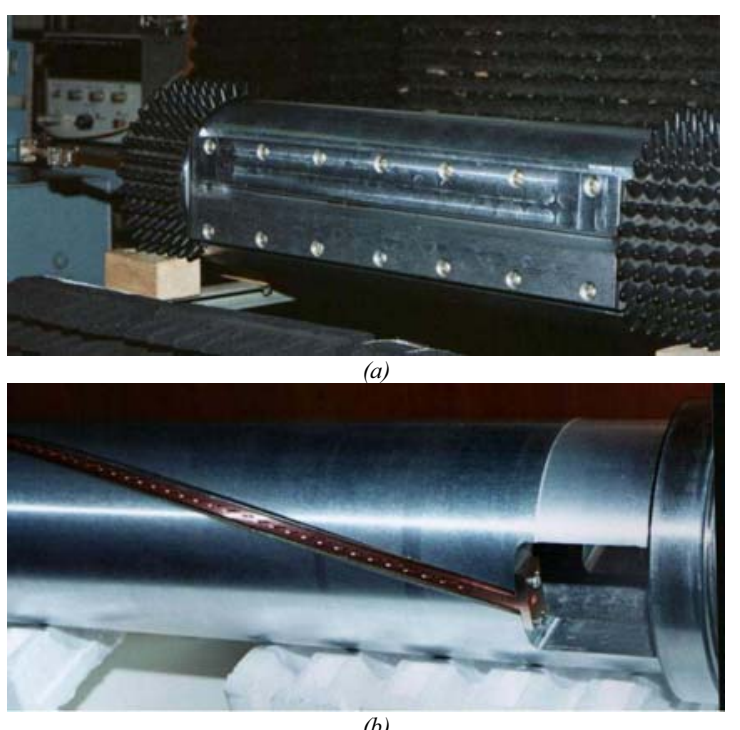

(b)

Figure 2 - Helical-Slot Synthetic Aperture Antenna: (a) General view and antenna stator; (b) Antenna rotor with resonant slots array in helical rectangular waveguide.

For $0.5 \mathrm{~m}$ range resolution one has to apply a radar signal with $300 \mathrm{MHz}$ bandwidth. This gives the following estimation for the time-bandwidth product: $\sim 10^{5}$, which provides rather good SNR for real-time imaging of the airport area of interest.

The second technical approach to realization of the suggested principle is the following. As a real aperture antenna, one has to use a waveguide with a not-radiating half-wavelength longitudinal slot in its wider wall (Figure 3). When covering this wall with a metallic tape having a half-wavelength transverse slot one provides condition for resonant radiation of the wave travelling inside the waveguide.

In order to enhance its efficiency one has to place a short circuit at the proper distance from the radiating slot. The tape may be self-connected into a ring and rotated with a given speed. Scanning rate depends on the tape ring rotating speed and also may be rather high provided a properly fast SAR processor. Unlike the previous case, this antenna does not need a rotary joint.

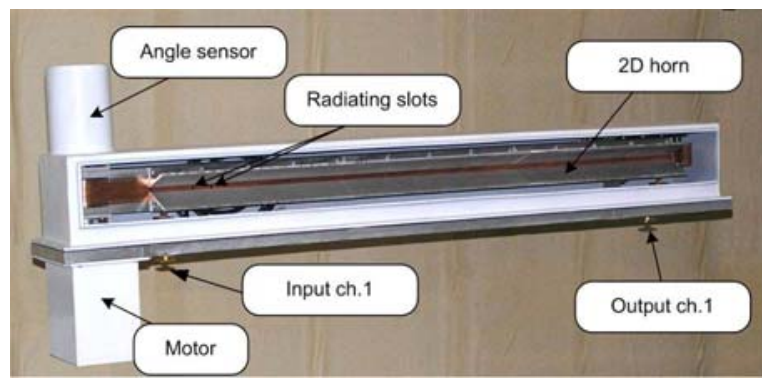

Figure 3 - Synthetic Aperture Antenna for Ka-band Ground Based SAR using motion of radiating slot along waveguide with open wall (the waveguide is beyond cooper tape).

We may use also step-like motion of the tape if we are not limited in time. This type of antenna has been also developed in LNDES NASU and tested jointly with Aerosensing Radar System, GmbH. One frame of the realtime video generated with the help of this antenna is presented in Figure 4. It is seen from Figure 4 that the antenna designed enables scanning of virtual antenna beam within azimuth sector of $\pm 65^{\circ}$. The tests carried out showed excellent results concerning its suitability for scanning antenna design on the basis of aperture synthesizing principle.

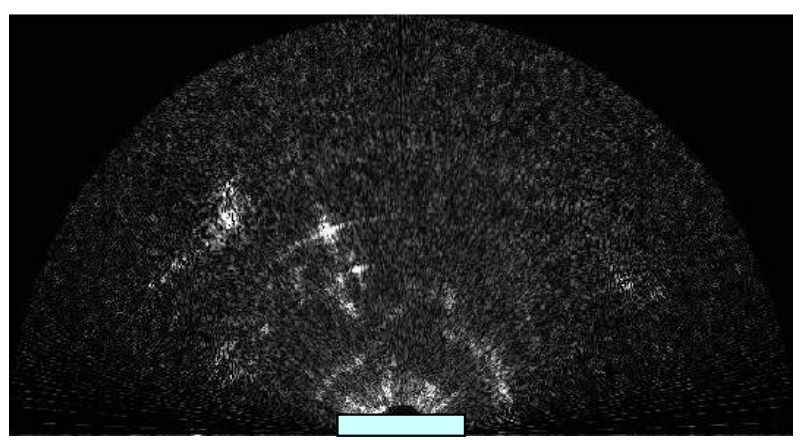

Figure 4 - Ka-band image obtained with the help of sliding slot antenna: viewing angle is about 130 degrees.

In the third type of scanning antennas a virtual shift of the radiating element is performed [7] via electro-mechanical switching of radiating elements which form a $1 \mathrm{D}$ array. The antenna made of a waveguide with a linear array of equally spaced resonant radiating slots. Each slot is shielded by a three-state-screening strip (TSSS) having three different states: (1) open, (2) close and (3) choke. The first/second state is used to open/close each radiating slot according to the control signal, while the third state is used to stop propagation of the feeding wave through the waveguide to enhance of the radiation efficiency. Flip-flop operation is to be implemented with help of electro-mechanical switches, 
e.g. combination of springs with electro-magnets. A linear virtual motion of the radiating slot is performed via switching of the TSSSs in the way enabling for each slot a sequential alternation of the following states: choke-state, open-state and close-state. The scanning rate of that antenna depends on switching time of the electro-mechanical TSSS. Similar design may be implemented for patch antennas. With this aim, one has to prepare a linear array of radiating patches at an upper row and choke patches at a lower row while feeding strip-line is to be placed between them. Each patch should be fed through a flip-flop switch. A linear virtual motion of the radiating patch is performed via connecting/disconnecting of the radiating patches and choke patches in the way similar to that in the previous case: (1) "radiating patch disconnected" and "choke patch connected"; (2) "radiating patch connected" and "choke patch disconnected", (3) "radiating patch disconnected" and "choke patch disconnected". For providing high efficiency, high decoupling of the radiating patches one has to use either MEMS switches having small losses, low power consumption and high rate isolation, or electromechanical flip-flop switches possessing similar performance. Design of SAA of the third type is in progress. We have shown potentialities of new type antenna, and specific choice of the SAA to be used in SMR depends on its required performance.

\section{NOISE RADAR TECHNOLOGY FOR SMR}

To validate the approach suggested the Ground Based Noise Waveform SAR (GB NW-SAR) has been developed [10] on the basis of the above second type antennas and NRT [8], [9], [11]. Its general view is shown in Figure 5.

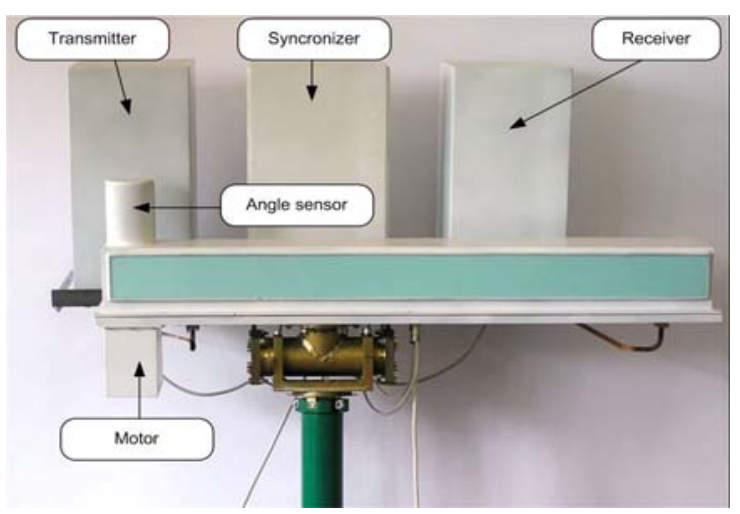

Figure 5 - General view of Ka-band Ground Based Noise Waveform SAR for near range surveillance.

Noise Waveform (NW) with a wide and smooth enough power spectrum, having fast decay of correlations enables to perform simultaneously the high rate signal compression, its optimal reception and minimization of range sidelobes. Besides, the ambiguity function of Noise Radar has no additional maximums, while for the periodical waveforms this is always the case. From that, particularly, follows that there is no contradictions for the NRT when providing the optimal conditions for simultaneous measurements of target distances and velocities; no theoretical limitations on the unambiguous working range; no needs in high peak power in the transmitted signals for a given working range. Besides, NR has excellent both LPI and EMC performance enabling its simultaneous operation within the same area. Main performance of the Ka-band SMR on the basis developed GB NW-SAR [10] is as follows: working range: $200 \mathrm{~m}-5000 \mathrm{~m}$; resolution cells $1.2 \mathrm{~m} \times 0.3 \mathrm{deg}$; average transmitted power: $(5-12) \mathrm{W}$; peak power $(10-24) \mathrm{W}$ for $50 \%$ duty cycle; antenna aperture $1 \mathrm{~m}$; full scan time: $0.05 \mathrm{~s}$. It is seen that a new concept enables design of SMR with essentially enhanced performance compared to conventional radar: no need in a rotating antenna; low level of transmitted power; high rate of radar image refreshment provided quasireal time SAR imaging. The latter is to be implemented on the basis of FPGA or Cell processor technologies.

The GB NW-SAR has been tested for short range imaging with off-line processing. The picture of the mapped area is presented in Figure 6, while its SAR image is shown in Figure 7.

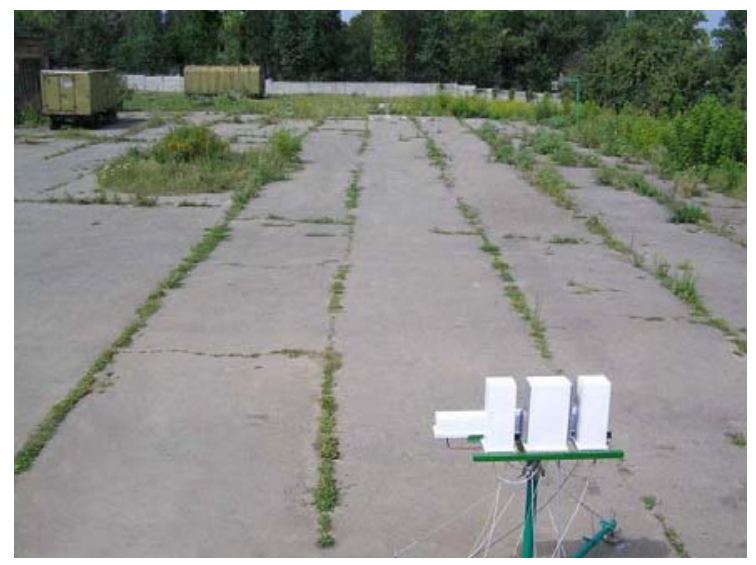

Figure 6 - Photograph of GB NW-SAR and mapped area.

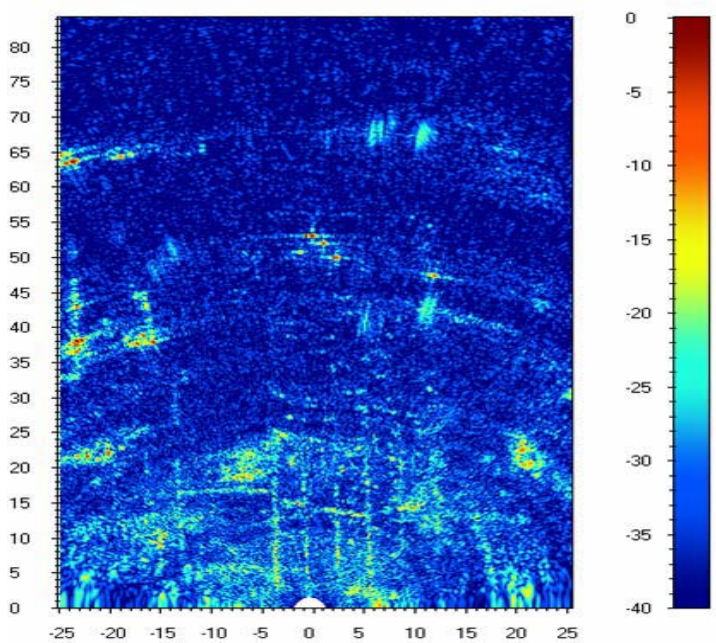

Figure 7 - SAR image of the area shown in Fig. 6 generated with the help of Ka-band GB NW-SAR

One may easily identify corner reflectors placed at 80 meters range; long cracks (along range) in asphalt surface and larger objects such as military trailers. Our experiments have shown capability of the GB NW-SAR to suppress clutter and residual fluctuations via increase in integration time which significantly improves SAR image quality. Since at different ranges, significantly different resolutions capabilities and transmitted power are required one may apply another concept for SMR design to enhance the SMR performance. Figure 8 shows coverage for one of possible 
options of such SMR implementation using suggested approach. A single SAA having a 42 degrees antenna pattern is used for surveillance/imaging of runway (a distant one: $\sim 5 \mathrm{~km}$ away) while the relatively short-range $(200 \mathrm{~m}-$ $2000 \mathrm{~m}$ ) area may be mapped using two other SAAs having 90 degrees antenna pattern each. After SAR/SAA processing the equivalent beam has equivalent width of 0.14 degrees provided by the $1.7 \mathrm{~m}$ synthesized aperture. Positions number of the equivalent beam equals 300 for runway surveillance radar, while for short-range area it will be about 1350 in total for both antennas.

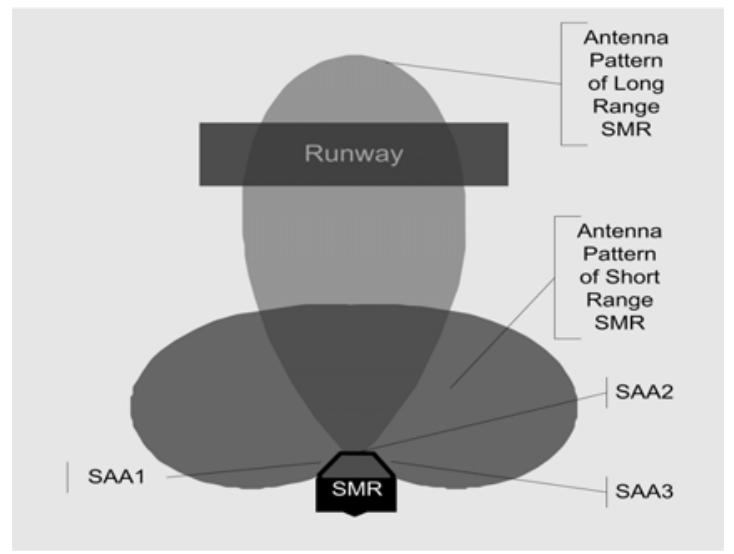

Figure 8 - Coverage of different areas of an airport using long range and short range SMRs and three Synthetic Aperture Antennae (SAA1, SAA2, SAA3).

In general, application of the suggested approach gives much more flexibility in SMR design unlike an application of a single rotating antenna SMR. The latter is in wide use now, but requires an upgrade since it has significant limitations in parameters such as integration time, MTI capability, clutter suppression, etc. and requires a high peak power and the application of a rotating antenna that complicates the SMR construction and maintenance.

\section{CONCLUSIONS}

Millimetre waves SMR have been suggested, designed and tested. Nevertheless there is a need for SMR performance enhancement. We have suggested a novel concept for SMR design based upon application of GB NW (Ground-Based Noise Waveforms) - SAR that uses antenna of a new type: Synthetic Aperture Antenna. Such SMR has excellent potential capabilities for design and development of compact, low energy consumption and cost effective SMR suitable, in particular, for monitoring of shadowed areas of airport layouts. An attractive feature of the novel approach suggested consists in the ability to produce a series of SAR images at so high rate, that it may be displayed as a realtime video. This is may be realized via fast motion of antenna radiator across the aperture and online SAR processing. This will permit enhanced surveillance in novel Advanced-Surface Movement Guidance and Control Systems.

\section{REFERENCES}

[1] G. Galati, M. Naldi, M. Ferri "Airport Surface Surveillance with a Network of Miniradars" IEEE
Trans. on Aerospace and Electronic System, Vol. 35, no.1, Jan. 1999, pp. 331-338.

[2] G. Galati, M. Ferri, M. Leonardi, P. Magarò, M. Gasbarra "A novel W-Band high resolution surface movement radar: operational trials, plot extraction and data processing" Proceedings IRS 2003, 30 Sept. - 2 Oct. Dresden, Germany, pp. 769-776.

[3] EUROCAE Working Group 41: "Surface Movement Guidance and Control Systems", ED-200, vol. 1-2, February 1994.

[4] G. Galati, P. Magarò, M. Leonardi, A. Cavallin "Data extraction in high resolution surface movement radar for A-SMCGS" Proceedings IRS 2004, 19-21 may 2004, Warsaw, Poland, pp. 245-250.

[5] M. Ferri, F. Serrano, V. Dainelli, G. Galati "Millimeter Wave Technologies for Airport Surface Movement Control Applications", $3^{\text {rd }}$ ESA Workshop on Millimetre Wave Technology and Applications 21-23 May 2003, Millilab, Espoo, Finland.

[6] K.A. Lukin, "A Novel Approach to Scanning Antenna Design", Proc. $4^{\text {th }}$ Int. Conf. on Antenna Theory and Techniques, Sevastopol, Ukraine, 2003, Vol. 11, pp. 290-293.

[7] K.A. Lukin, "Sliding Antennas for Noise Waveform SAR", Applied Radio Electronics, 2005, Vol. 4, \#1, pp. 103-106.

[8] K.A. Lukin, "Noise Radar Technology", Radiophysics and Electronics, Kharkiv, Institute for Radiophysics and Electronics of NASU, 1999, vol.4, \#. 3, pp.105-111 (In Russian). Translation in Telecommunications and Radio Engineering, V.55, \#12, 2001, pp.8-16.

[9] K.A. Lukin, "Noise Radar Technology: the Principles and Short Overview", Applied Radio Electronics, 2005, Vol. 4, \#1, pp. 4-13.

[10] K.A. Lukin. "Ground Based Noise-Waveform-SAR for Monitoring of Chernobyl Sarcophagus", Proceedings of Int. Radar Symposium, IRS-2005, 06-08 September 2005, Berlin, Germany, pp. 655-659.

[11] M. Ferri, G. Galati, G. Pavan, "W-band Noise Radar in Short Range Applications" Proceedings IRS 2008, 2023 May 2008, Wroclaw, Poland, pp. 211-214.

[12] K.A. Lukin, "Capability of Noise Radar Technology in Design for Airport Surveillance Sensors" Proc. of JISSA'2001, Paris, Dec.11-12, 2001, pp.23-32.

[13] A. De Fazio, M. Ferri, G. Galati., M. Leonardi, "High Resolution, Millimetre-Wave Radar Techniques for ASMGCS: Pre-Operational Evaluation and Technical Developments" IEEE International Conference RADAR 2004, Toulouse (France) 18-22 October 2004.

\section{Main Menu}

